\title{
Deep Whole Genome Sequencing Identifies Recurrent Genomic Alterations in Commonly-Used Breast Cancer Cell Lines and Patient Derived Xenograft Models
}

\section{Niantao Deng}

Garvan Institute of Medical Research

Andre Minoche

Garvan Institute of Medical Research

Kate Harvey

Garvan Institute of Medical Research

Meng Li

University of California San Francisco

Juliane Winkler

University of California San Francisco

Andrei Goga

University of California San Francisco

Alex Swarbrick ( $\sim$ a.swarbrick@garvan.org.au )

Garvan Institute of Medical Research https://orcid.org/0000-0002-3051-5676

Research article

Keywords: Breast Cancer Cell Lines, Patient Derived Xenografts, Whole Genome Sequencing, Structural Variants, Non-coding Mutations

Posted Date: September 30th, 2021

DOI: https://doi.org/10.21203/rs.3.rs-859624/v2

License: (9) This work is licensed under a Creative Commons Attribution 4.0 International License. Read Full License 


\section{Title page}

2 Deep whole genome sequencing identifies recurrent genomic alterations in

3 commonly-used breast cancer cell lines and patient derived xenograft models

$5 \quad$ List the full names, institutional addresses and email addresses for all authors

6 Niantao Deng ${ }^{1,2}$, Andre Minoche ${ }^{2,3}$, Kate Harvey ${ }^{1}$, Meng Li $^{4,5}$, Juliane Winkler ${ }^{4,5}$,

$7 \quad$ Andrei Goga ${ }^{4,5}$, Alex Swarbrick ${ }^{1,2}$

$8{ }^{1}$ The Kinghorn Cancer Centre \& Cancer Research Theme, Garvan Institute of

9 Medical Research, Sydney, Australia; ${ }^{2}$ St Vincent's Clinical School, Faculty of

10 Medicine, UNSW, Australia; ${ }^{3}$ Kinghorn Centre for Clinical Genomics, Garvan

11 Institute of Medical Research, Sydney, Australia; ${ }^{4}$ Department of Cell \& Tissue

12 Biology, University of California, San Francisco, San Francisco, CA, USA;

$13{ }^{5}$ Department of Medicine, University of California, San Francisco, San Francisco, 14 CA, USA.

\section{Abstract}

17 Background: Breast cancer cell lines (BCCLs) and patient-derived xenografts (PDX)

18 are the most frequently used models in breast cancer research. Despite their

19 widespread usage, genome sequencing of these models is incomplete, with previous

20 studies only focusing on targeted gene panels, whole exome or shallow whole genome

21 sequencing. Deep whole genome sequencing is the most sensitive and accurate

22 method to detect single nucleotide variants and indels, gene copy number and

23 structural events such as gene fusions.

24 Results: Here we describe deep whole genome sequencing (WGS) of commonly used

25 BCCL and PDX models using the Illumina X10 platform with an average $\sim 60 \mathrm{x}$ 
coverage. We identify novel genomic alterations, including point mutations and

27 genomic rearrangements at base-pair resolution, compared to previously available

28 sequencing data. Through integrative analysis with publicly available functional

29 screening data, we annotate new genomic features likely to be of biological

30 significance. CSMD1, previously identified as a tumor suppressor gene in various

31 cancer types, including head and neck, lung and breast cancers, has been identified

32 with deletion in 50\% of our PDX models, suggesting an important role in aggressive

33 breast cancers.

34 Conclusions: Our WGS data provides a comprehensive genome sequencing resource

35 of these models.

$37 \quad-194$ words

38 Keywords

39 Breast Cancer Cell Lines, Patient Derived Xenografts, Whole Genome Sequencing,

40 Structural Variants, Non-coding Mutations

\section{Background}

43 Breast cancer cell line (BCCL) models are indispensable tools to study breast cancer

44 biology and heterogeneity. Molecular profiling of BCCLs has generated useful

45 insights into breast cancer subtypes and provides a resources for cancer gene

46 discovery (Kao et al., 2009; Neve et al., 2006). A number of large-scale cancer cell

47 line projects have characterised hundreds of cell lines with whole transcriptome

48 profiling (Klijn et al., 2015), DNA microarray and targeted sequencing (Barretina et

49 al., 2012). These studies provide great resources for cancer cell line studies, however,

50 none of them have performed deep whole genome-wide sequencing analysis of 
51 genetic changes, including mutations and structural variations, on these critical breast

52 cancer models.

53 Patient derived xenograft (PDX) models, which closely resemble the heterogeneity of

54 clinical BC, are established as important preclinical models (Bruna et al., 2016;

55 DeRose et al., 2011). These breast cancer PDX models have been only characterised

56 by shallow $(<1 \mathrm{X}$ coverage) whole genome sequencing (WGS) or exome sequencing

57 (Bruna et al., 2016).

58 WGS is the most sensitive method for detecting structural variants (SVs) and copy

59 number variants (CNVs) and the only method to survey non-coding mutations. WGS

60 is also a more powerful tool compared to exome-seq in detecting exome variants

61 (Belkadi et al., 2015). To fully understand the genomic features of these models, it is

62 important to conduct deep WGS analysis of these models to exhaustively identify

63 complex genomic features of PDX models. In this study, we performed deep WGS to

64 provide a comprehensive resource of genomic events of these important BCCLs and

65 PDX models.

66

$67 \quad$ Results

68 Whole genome sequencing of breast cancer cell line models

69 According to a PubMed search, the top six studied BCCLs are MCF7, MDAMB231,

70 T47D, SKBR3, MCF10A and MDAMB468, which cover more than 90\% of all BCCL

71 associated studies across more than 70,000 publications (Figure S1). Previous studies

72 on these cell lines are restricted to whole-exome or low-pass whole genome

73 sequencing at $0.2 \mathrm{X}$ coverage (Ben-David et al., 2018). Here we describe whole

74 genome sequencing (WGS) of these models on the Illumina X10 platform with an

75 average $\sim 60 \mathrm{x}$ coverage, including two replicates of the most-commonly used MCF7 
cell line $(\sim 53 \mathrm{x}$ and $\sim 78 \mathrm{x})$. Raw reads were mapped to human genome GRCh37 and single nucleotide variants were called using the Issac pipeline (Raczy et al., 2013). WGS is the most accurate way to assess cell line identity (Yu et al., 2015). We have compared the genotyping calls from our WGS data with SNP array from two independent studies previously published (Barretina et al., 2012; Heiser et al., 2012). Correlation analysis of the genotyping calls at the same genomic loci show high concordance between ours and the published data $(\mathrm{R}>0.9)$ (Figure $\mathrm{S} 2)$, confirming the identity of all cell lines used in this study. In total, we have identified 3,540,312 to 4,108,844 variants per sample from the WGS data, including SNVs and small indels (Table S1). The majority of variants are present in the dbSNP database (Sherry et al., 2001) $(94.0 \% \sim 96.2 \%)$ (Table S1). About $90 \%$ of these dbSNP variants have also been reported in the 1000 genome projects (Genomes Project et al., 2012), suggesting most variants in these cell lines models represents common variants in the human population, similar to the previous finding of WGS of the Hela cell line (Landry et al., 2013). However, each of the cell lines has about $5 \%$ of total variants that are cell line specific variants (Figure 1A).

In MCF7, for example, more than half of the cell line specific variants are located in inter-genic regions and $\sim 1 / 3$ are found in introns (Figure 1B). We compared the list of missense mutation in MCF7 to a recent study of multiple strains of MCF7s (BenDavid et al., 2018) (Figure 1C). Most of the missense mutations in (Ben-David et al., 2018) shared between MCF7 strains, therefore likely to come from the founder tumor, were have been identified in this study (20/23), and the concordance with our data increases as the number of mutant MCF7 strains increases (Figure 1D.\& 1E). This suggests that the large majority of variants we have identified are real. In addition to the mutations reported in Ben-David et al, our WGS data identified 9,555 additional 
101

124 In addition to SVs, we also identified copy number alterations in these cell line 125 models (Table S5). WGS has improved accuracy in detecting CNVs compared to

missense mutations, 635 of which are not reported in 1000 genome or dbSNP database (Table S2). Sequencing of large cohorts of breast cancer tissue has revealed recurrent mutations in long non-coding genes including MALAT1 and NEAT1(NikZainal et al., 2016). Among all the cancer associated long non-coding genes reported (Quek et al., 2015) ((Gao et al., 2021), Lnc2Cancer v3.0), we identified mutations of MALAT1, HOTAIR and ZFAS1 in these cell line models. For example, Malat1 showed a heterozygous mutation (chr11:65271832 T $>$ C) in MCF7, but not in MDA-MB-231 (Figure S3). The complete list of variants in the non-coding regions in these cell lines models (Table S3) could serve as a useful database for selection of models in noncoding RNA studies.

In addition to the SNVs, we also called SVs and CNVs from the WGS data using different methods, including Breakdancer (Chen et al., 2009) and Delly (Chen et al., 2009) for structural variants; CNVnator (Abyzov, Urban, Snyder, \& Gerstein, 2011) and Lumpy (Abyzov et al., 2011) for copy number (see Methods). A summary of SVs and CNVs identified in MCF7 and the other four cell lines is shown in Figure 2A and Figure S4 respectively. In total, we have identified 321 inter-chromosomal SVs, with $38 \sim 108$ SVs per cell line (Table S4). We use the GREAT program (McLean et al., 2010) to perform pathway analysis of the SVs events from individual cell lines. As expected, the top enriched pathways are genes in amplified regions previously identified from breast cancer, such as genes like ZNF217 and BCAS3 in MCF7 (Hampton et al., 2009); a selected list of luminal genes have also been enriched in the luminal cell lines MCF7 and T47D (Figure S5). 
exome-seq due to its uniform coverage (Belkadi et al., 2015). We compared the

127 CNVs identified from MCF7 with existing data from array-based COSMIC and

128 shallow WGS from Ben-David et al (Figure 2B, Table S6). There is a poor overlap

129 between this study and COSMIC, however, a substantial proportion (138/478) of the

130 CNVs discordant between this study and COSMIC are reported in Ben-David et al

131 using shallow WGS, suggesting the array-based method missed a lot of CNVs due to

132 poor coverage. WGS can identify the known key copy number events in each of the

133 cell lines with fine resolution, including AURKA and MYC amplification, CDKN2A

134 (Figure 2C) deletion in MCF7, ERBB2 amplification in SKBR3, and CDKN2A

135 deletion in MDAMD231 (Table S3). Our WGS data can also identify novel CNVs not

136 reported in either COSMIC or Ben-David et al. LINC00290, a long non-coding RNA,

137 which has been previously reported to undergo copy number loss in a pan-cancer

138 study (Zack et al, 2013), has a homozygous deletion in MCF7 (Figure 2D).

139 Furthermore, CNV boundaries were accurately detected by WGS compared to WES,

140 in many cases with base pair precision (Table S5). For example, in MCF7 cell line,

141 the boundaries of the homozygous deletion cover only CDKNA2A, and the nearby

142 CDKN2B is in a hemizygous deletion region (Figure 2C). FOXA1 copy number gain

143 is in a focal amplicon, whereas GATA3 is in a broad copy number gain region about

$1440.5 \mathrm{Mb}$ long \& ESR1 copy number gain is towards the 5' end of the gene. (Figure S6).

145 In order to estimate the functional impact of these variants, we compared our list of

146 copy number variations with functional screen data from these cell lines (Marcotte et

147 al., 2016). In MCF7, 33 out of the 445 copy number events harbor at least one of the

148 breast cancer essential genes from (Marcotte et al., 2016). Interestingly this reveals

149 coordinated amplification of ESRI and its co-factors NCOA3 and GATA3 and pioneer

150 factor $F O X A 1$, perhaps explaining the extreme estrogen-sensitivity of this cell line 
151 (Table S5). Therefore, this study resolves known copy number events at nucleotide

152 resolution and reports a substantial number of new copy number variants, some with

153 evidence for function in these commonly-used cell line models.

154

155

156

157

158

159

160

161

162

163 the reads mappable to human genome, we identified 3,435,230 to 4,172,800 variants

164 per sample from the WGS data, including SNVs and small indels (Table S1).

165 Similarly to the cell line data, the majority have been identified in the dbSNP database

166 (Sherry et al., 2001) (95.6\% 95.9\%) (Table S1). About $84 \%$ of these variants

167 identified in dbSNP have also been reported in 1000 genome projects (Genomes

168 Project et al., 2012). Each of the PDXs has about 4\% of total variants as PDX specific

169 variants (Figure 3A). In HCI002, more than half of the PDX line specific variants are

170 located in inter-genic regions, followed by variants in introns (Figure 3B). To validate

171 our findings, we performed exome sequencing analysis of selected models and

172 identified There are 12 non-synonymous mutations identified using an independent

173 exome sequencing analysis of these models, all of which have been also identified in

174 our WGS data (Table S8). 
175 Using a stringent cutoff (Minoche et al., 2021), we have created a list of high

176 confidence copy number alterations and structural variants for each of the PDXs

177 (Table S9\&S10). Recurrently amplified regions across the PDX models are

178 chromosome 8q in HCI002/005/010/012 (covering MYC, Table S11) and

179 chromosome 7p in HCI004/HCI008 (covering EGFR). Frequent deleted regions

180 harbor classic tumour suppressor genes, for example PTEN in HCI004/HCI010

181 (Figure 3C, Table S9).

182 Because PDX tumours probably model more aggressive breast cancers (DeRose et

183 al), we proposed that genomic analysis of PDX models may reveal genes associated

184 with very aggressive disease. Interestingly, the most frequently deleted gene in PDX

185 models, CSMD1(in 3 out of our six PDX models), is much more frequently deleted in

186 metastatic breast cancer (14\%) than in early disease (4\%) (Figure 4A). Low

187 expression of CSMD1 is also associated with poor survival outcome in the

188 METABRIC early cancer cohort (Figure 4B). Interestingly, we identified copy

189 number loss of CSMD1 in 23 of 32 PDXs in another PDX sequencing dataset (Bruna

190 et al., 2016). These data suggest that CSMD1 plays a critical role in suppressing

191 growth or survival of metastatic breast cancers.

193 Discussion

194 We have completed whole genome analysis for widely used breast models, including

195 mutations, copy number and SVs. The data has refined previously finding in cell lines

196 such as $A U R K A$ and $M Y C$ amplification/CDKN2A deletion in MCF7, ERBB2

197 amplification in SKBR3, and CDKN2A deletion in MDAMD231 at nucleotide

198 resolution. Our WGS data also identified novel mutations in non-coding genes and

199 novel CNVs such as a homozygous deletion in LINC00290, and will be an important 
resource for research in this area. Since WGS is far more accurate in identifying

201 CNVs boundaries, it can provide further insights into well-known cancer associated

202 genes, such as focal amplification of FOXA1 in MCF7 versus broad copy number

203 gain in GATA3 and partial gain of ESR1. By comprehensively identifying the genomic

204 features of these models, the field can now choose appropriate models to examine the

205 functional significance of genes or pathways of interest.

206 Genomic studies of PDX may help us identify genomic features associated with very

207 poor prognosis, as growth in a PDX acts as a 'filter' to enrich for the most aggressive

208 tumours and cells that otherwise occur at low patient frequency in unselected breast

209 cancer cell populations. In support of this, we found recurrent deletion of CSMD1 in

210 the PDXs models and its associated with metastatic disease and survival. Further

211 extension of this idea to more PDX models may reveal further drivers of aggressive

212 disease. The WGS data in this study is a valuable resource for other genomics studies

213 to map to, for instance, CHIP-Seq or RNA-Seq studies to focus on non-coding and

214 regulatory regions.

216 Conclusions

217 We have applied deep whole genome sequencing (WGS) of commonly used cell lines

218 and PDX models using the Illumina X10 platform with an average $\sim 60 \mathrm{x}$ coverage.

219 We show that this resource can be used to identify novel genomic alterations,

220 including point mutations and genomic rearrangements, compared to previously

221 available sequencing data.

222 Specific outcomes include:

223 - A comprehensive list of point mutations, copy number and structural variants

224 for cell lines and PDX models.

225 - Integrative analysis with publicly available functional screening data identifies

226 new genomic features of biological significance. 
- CSMD1, a known tumour suppressor gene, identified with deletion in $50 \%$ of our PDX models, suggesting an important role in aggressive breast cancers.

\section{Methods}

232 Sample acquisition and preparation

233 The MCF7 cell line was originally obtained from Michigan Cancer Foundation, the

234 MCF10A from Brugge Lab, Boston, the MDAMB231 from EG\&G Mason RI

235 Worcester MA, and the MDAMB468, SKBR3 and T47D were from ATCC. Cells

236 were cultured in their standard media to expand and frozen cell pellets used for DNA

237 extraction. Breast Cancer PDX models were obtained from Alana Welm, and were

238 expanded in house using techniques previously reported in DeRose et al 2010.

\section{DNA extraction}

241 The DNeasy Blood \& Tissue Kit (Qiagen) was used for DNA isolation from about

$24225 \mathrm{mg}$ frozen tumor, according to the manufacturer's recommendations. RNase A

243 (Qiagen) was used to obtain RNA-free genomic DNA. Only isolated DNA with

244 A260/280 and A260/230 ratios above 1.8 and proven to be high quality by gel

245 electrophoresis were used for sequencing.

247 Whole Genome Sequencing analysis of cell lines and PDX

248 The five cancer cell lines MCF7, MDAMB231, T47D, SKBR3, MDAMB468 and one

249 non-malignant cell line MCF10A and six PDX models were submitted for sequencing 250 with at least $60 x$ coverage. Raw reads were mapped to human genome GRCh37 using

251 Issac aligner from Illumina and called point mutations and small indels by Issac.

252 Copy number alterations from the cell lines were estimated from cn.mops ( $R$ 
package), and CNVnator (Abyzov et al., 2011). Structural variants were computed

254 from Breakdancer (Chen et al., 2009), Delly (Chen et al., 2009) and Lumpy (Abyzov

255 et al., 2011). Structural variants were annotated and visualized using ClinSV

256 (Minoche et al., 2021) and IGV (Robinson et al., 2011). Raw sequencing files (fastqs)

257 and variants (vcfs) are deposited at European Genome Archive. Data access will need 258 to be submitted Data Access Committee EGAC00001001974.

260 Cell line identity check using SNP array

261 These common cell lines have been profiled by Affymetrix SNP 6.0 array previously

262 in multiple studies. Raw cel files were downloaded from the Heiser et al and CCLE

263 and were analysed by affymetrix genotyping console software. Correlation analysis

264 between the genotyping calls between array and WGS data to confirm cell line

265 identity.

266

267 Ethical Approval and Consent to participate

268 All required ethic approvals for investigations of the patient derived xenograft mouse 269 models have been received for A/P Alex Swarbrick's lab.

271 Consent for publication

272 All authors have read the manuscript and provided consent for publication.

273

274 Availability of supporting data

275 All data analysed in this study are included in this article and its supplementary

276 information files. The raw sequencing data supporting these findings are available at 
European Genome Archive (Data access will need to be submitted Data Access

Committee EGAC00001001974).

\section{Competing interests}

281 The authors declare that they have no competing interests.

282

283

\section{Funding}

284 This study is supported by a postdoctoral fellowship from the National Breast Cancer 285 Foundation of Australia to N.D. and a Senior Research Fellowship from the NHMRC to AS. J.W is supported by an EMBO long-term post-doctoral fellowship. We acknowledge the support of The Petre Foundation, the Estate of the late RT Hall, Deborah and John Mcmurtrie and the Love Your Sister Foundation. We thank Alana Welm for providing the PDX models.

\section{Authors' contributions}

292 N.D and A.S conceived the experiment design and wrote the manuscript. N.D. and 293 A.M. analysed the sequencing data. K.H. prepared the samples for whole genome 294 sequencing. M.L, J.W, A.G. provided data for mutational validations in PDXs.

\section{Acknowledgements}

297 We thank Gillian Lehrbach for assistance with cell culture and Jess Yang, Andrea

298 McFarland and Nicola Forman for PDX experimentation.

\section{References} approach to discover, genotype, and characterize typical and atypical 
CNVs from family and population genome sequencing. Genome Res, 21(6), 974-984. doi:10.1101/gr.114876.110

Barretina, J., Caponigro, G., Stransky, N., Venkatesan, K., Margolin, A. A., Kim, S., . . Garraway, L. A. (2012). The Cancer Cell Line Encyclopedia enables predictive modelling of anticancer drug sensitivity. Nature, 483(7391), 603-607. doi:10.1038/nature11003

Belkadi, A., Bolze, A., Itan, Y., Cobat, A., Vincent, Q. B., Antipenko, A., ... Abel, L. (2015). Whole-genome sequencing is more powerful than whole-exome sequencing for detecting exome variants. Proc Natl Acad Sci U S A, 112(17), 5473-5478. doi:10.1073/pnas.1418631112

Ben-David, U., Siranosian, B., Ha, G., Tang, H., Oren, Y., Hinohara, K., . . Golub, T. R. (2018). Genetic and transcriptional evolution alters cancer cell line drug response. Nature, 560(7718), 325-330. doi:10.1038/s41586-018-0409-3

Bruna, A., Rueda, O. M., Greenwood, W., Batra, A. S., Callari, M., Batra, R. N., . . . Caldas, C. (2016). A Biobank of Breast Cancer Explants with Preserved Intra-tumor Heterogeneity to Screen Anticancer Compounds. Cell, 167(1), 260-274 e222. doi:10.1016/j.cell.2016.08.041

Chen, K., Wallis, J. W., McLellan, M. D., Larson, D. E., Kalicki, J. M., Pohl, C. S., . . . Mardis, E. R. (2009). BreakDancer: an algorithm for high-resolution mapping of genomic structural variation. Nat Methods, 6(9), 677-681. doi:10.1038/nmeth.1363

DeRose, Y. S., Wang, G., Lin, Y. C., Bernard, P. S., Buys, S. S., Ebbert, M. T., . . Welm, A. L. (2011). Tumor grafts derived from women with breast cancer authentically reflect tumor pathology, growth, metastasis and disease outcomes. Nat Med, 17(11), 1514-1520. doi:10.1038/nm.2454

Gao, Y., Shang, S., Guo, S., Li, X., Zhou, H., Liu, H., .. Z Zhang, Y. (2021). Lnc2Cancer 3.0: an updated resource for experimentally supported IncRNA/circRNA cancer associations and web tools based on RNA-seq and scRNA-seq data. Nucleic Acids Res, 49(D1), D1251-D1258. doi:10.1093/nar/gkaa1006

Genomes Project, C., Abecasis, G. R., Auton, A., Brooks, L. D., DePristo, M. A., Durbin, R. M., ... McVean, G. A. (2012). An integrated map of genetic variation from 1,092 human genomes. Nature, 491(7422), 56-65. doi:10.1038/nature11632

Hampton, O. A., Den Hollander, P., Miller, C. A., Delgado, D. A., Li, J., Coarfa, C., . . . Milosavljevic, A. (2009). A sequence-level map of chromosomal breakpoints in the MCF-7 breast cancer cell line yields insights into the evolution of a cancer genome. Genome Res, 19(2), 167-177. doi:10.1101/gr.080259.108

Heiser, L. M., Sadanandam, A., Kuo, W. L., Benz, S. C., Goldstein, T. C., Ng, S., .. . Spellman, P. T. (2012). Subtype and pathway specific responses to anticancer compounds in breast cancer. Proc Natl Acad Sci U S A, 109(8), 2724-2729. doi:10.1073/pnas.1018854108

Kao, J., Salari, K., Bocanegra, M., Choi, Y. L., Girard, L., Gandhi, J., ... Pollack, J. R. (2009). Molecular profiling of breast cancer cell lines defines relevant tumor models and provides a resource for cancer gene discovery. PLoS One, 4(7), e6146. doi:10.1371/journal.pone.0006146

Klijn, C., Durinck, S., Stawiski, E. W., Haverty, P. M., Jiang, Z., Liu, H., . . Zhang, Z. (2015). A comprehensive transcriptional portrait of human cancer cell lines. Nat Biotechnol, 33(3), 306-312. doi:10.1038/nbt.3080 
Landry, J. J., Pyl, P. T., Rausch, T., Zichner, T., Tekkedil, M. M., Stutz, A. M., ... Steinmetz, L. M. (2013). The genomic and transcriptomic landscape of a HeLa cell line. G3 (Bethesda), 3(8), 1213-1224. doi:10.1534/g3.113.005777

Marcotte, R., Sayad, A., Brown, K. R., Sanchez-Garcia, F., Reimand, J., Haider, M., .. . Neel, B. G. (2016). Functional Genomic Landscape of Human Breast Cancer Drivers, Vulnerabilities, and Resistance. Cell, 164(1-2), 293-309. doi:10.1016/j.cell.2015.11.062

McLean, C. Y., Bristor, D., Hiller, M., Clarke, S. L., Schaar, B. T., Lowe, C. B., . . Bejerano, G. (2010). GREAT improves functional interpretation of cisregulatory regions. Nat Biotechnol, 28(5), 495-501. doi:10.1038/nbt.1630

Minoche, A. E., Lundie, B., Peters, G. B., Ohnesorg, T., Pinese, M., Thomas, D. M., . . Cowley, M. J. (2021). ClinSV: clinical grade structural and copy number variant detection from whole genome sequencing data. Genome Med, 13(1), 32. doi:10.1186/s13073-021-00841-x

Neve, R. M., Chin, K., Fridlyand, J., Yeh, J., Baehner, F. L., Fevr, T., ... Gray, J. W. (2006). A collection of breast cancer cell lines for the study of functionally distinct cancer subtypes. Cancer Cell, 10(6), 515-527. doi:10.1016/j.ccr.2006.10.008

Nik-Zainal, S., Davies, H., Staaf, J., Ramakrishna, M., Glodzik, D., Zou, X., . . . Stratton, M. R. (2016). Landscape of somatic mutations in 560 breast cancer whole-genome sequences. Nature, 534(7605), 47-54. doi:10.1038/nature17676

Quek, X. C., Thomson, D. W., Maag, J. L., Bartonicek, N., Signal, B., Clark, M. B., . . Dinger, M. E. (2015). lncRNAdb v2.0: expanding the reference database for functional long noncoding RNAs. Nucleic Acids Res, 43(Database issue), D168-173. doi:10.1093/nar/gku988

Raczy, C., Petrovski, R., Saunders, C. T., Chorny, I., Kruglyak, S., Margulies, E. H., . . Tanner, S. W. (2013). Isaac: ultra-fast whole-genome secondary analysis on Illumina sequencing platforms. Bioinformatics, 29(16), 2041-2043. doi:10.1093/bioinformatics/btt314

Robinson, J. T., Thorvaldsdottir, H., Winckler, W., Guttman, M., Lander, E. S., Getz, G., \& Mesirov, J. P. (2011). Integrative genomics viewer. Nat Biotechnol, 29(1), 24-26. doi:10.1038/nbt.1754

Sherry, S. T., Ward, M. H., Kholodov, M., Baker, J., Phan, L., Smigielski, E. M., \& Sirotkin, K. (2001). dbSNP: the NCBI database of genetic variation. Nucleic Acids Res, 29(1), 308-311. Retrieved from http://www.ncbi.nlm.nih.gov/pubmed/11125122

Yu, M., Selvaraj, S. K., Liang-Chu, M. M., Aghajani, S., Busse, M., Yuan, J., . . . Neve, R. M. (2015). A resource for cell line authentication, annotation and quality control. Nature, 520(7547), 307-311. doi:10.1038/nature14397 
Figure 1: Summary of single nucleotide variations (SNVs) in six breast cancer cell

397 lines, including two replicates for MCF-7. (A) Classification of SNVs into different 398 categories: overlap with 1000 genome project; overlap with dbSNP but not in 1000 399 genome project; cell-line specific events; (B) Distribution of the SNVs in MCF-7 in 400 respect to location of protein coding genes. (C) unsupervised hierarchical clustering 401 of MCF7 in this study together with 27 MCF7 strains in Ben-David et al, based on 402 their missense mutation obtained from supplementary information from (Ben-David 403 et al) (D) Percentage of overlapping missense mutations between our findings and 404 those identified in multiple strains of MCF7s in Ben-David et al. (E) Venn diagram 405 shows the overlap of missense mutations in Ben-David et al and this study.

407 Figure 2: (A) Genomics landscape of copy number and structural variants in six 408 breast cancer cell lines. Circos plots of each individual cell lines are included. Copy 409 number events are summarized in the inner circle with red and blue color indicates 410 copy number gains and blue respectively, two inner circles are represented two 411 replicates of MCF7 samples (one 30X and one 60X). Arcs connecting two loci of 412 difference chromosomes indicate inter-chromosomal structural variations. (B) Venn 413 diagram shows the overlap of CNV genes between COSMIC, Ben-David et al and

414 this study. (C) Representative genome browser view of copy number alterations 415 covering a common CNV gene, CDKN2A. Tracks from top to bottom: depth of 416 coverage in an NA12878 control (control), all reads in the sample (all reads), or reads 417 with mapping quality $>=20(\mathrm{MQ}>20)$, the average mapping quality of aligned reads 418 from the sample (MQ, if no reads align $\mathrm{MQ}=0$ ), coverage standard deviation from 500 419 controls (Coverage SD, indicating common CNV), overlapping segmental 420 duplications published by Bailey JA et al. 2002 (SEG-DUP, used as control for 
421 germline CNVs), discordant pairs (DP), split reads (SR), variants from the Database

422 of Genomic Variants (DGV), and RefSeq genes (Genes). (D) Representative genome

423 browser view plot of a novel CNV gene LINC00290 in this study.

425 Figure 3: Summary of SNVs in breast cancer patient-derived xenograft models: (A)

426 classifications of SNVs into three categories: overlap with 1000 genome project;

427 overlap with dbSNP but not in 1000 genome project; PDX-specific variants. (B)

428 Distribution of the SNVs in HCI002 model in respect to location of protein coding

429 genes. (C) Representative genome browser view of copy number alteration covering

430 PTEN in HCI004 model. Tracks from top to bottom: depth of coverage in HCI004

431 germline (control) and HCI004 PDX, and the average mapping quality of aligned

432 reads from the sample (MQ, if no reads align $\mathrm{MQ}=0)$, coverage standard deviation

433 from 500 controls (Coverage SD, indicating common CNV), overlapping segmental

434 duplications published by Bailey JA et al. 2002 (SEG-DUP, used as control for

435 germline CNVs), discordant pairs (DP), split reads (SR), variants from the Database

436 of Genomic Variants (DGV), and RefSeq genes (Genes).

438 Figure 4: Genes with enriched genomic alterations in PDXs

439 (A,B) Genes with frequent copy number alterations in PDXs samples showing higher 440 copy number variations in a metastatic breast cancer cohort than a primary breast

441 cancer cohort (cbioportal). Each row indicates a gene and each column indicates a 442 breast cancer sample. Copy number gains are in red and deletions are in blue.

443 CSMD1, for example, showing a much high frequency of copy number deletion in the 444 metastatic cohort. (C) Kaplan-Meier survival analysis of METABRIC samples 
445 stratified by CSMD1 expression status. Top 25\% samples with high CSMD1 is in red, 446 showing a better survival outcome compared to those with low CSMD1 expression. 447 
Figure 1

A

B

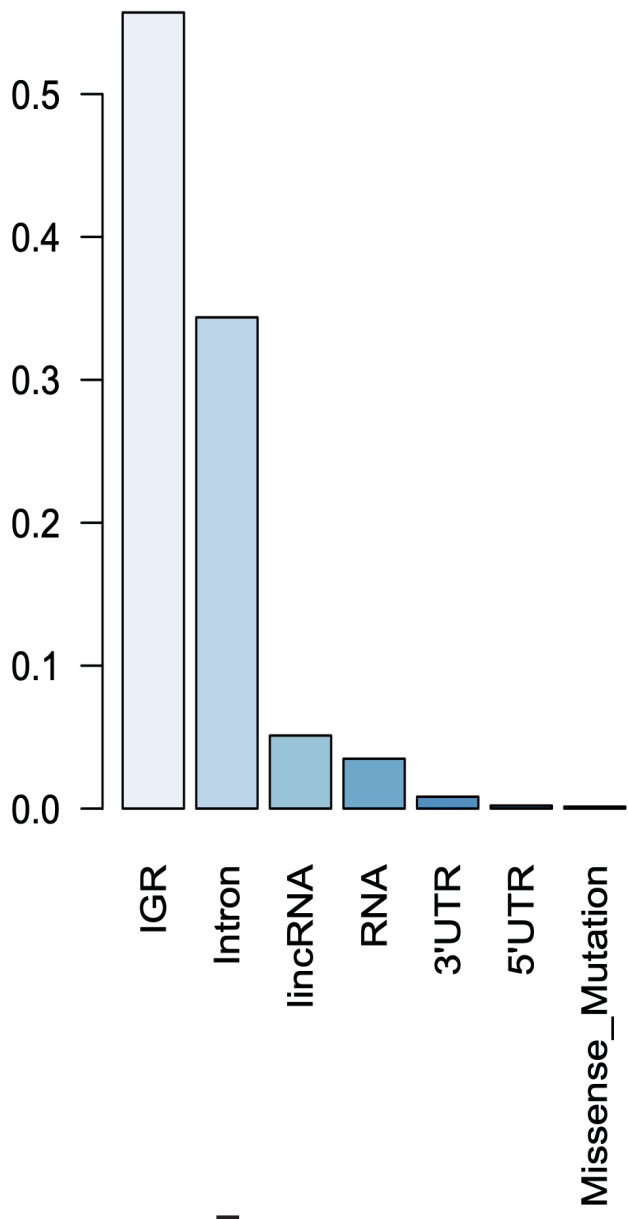

C

$\mathrm{D}$

$E$
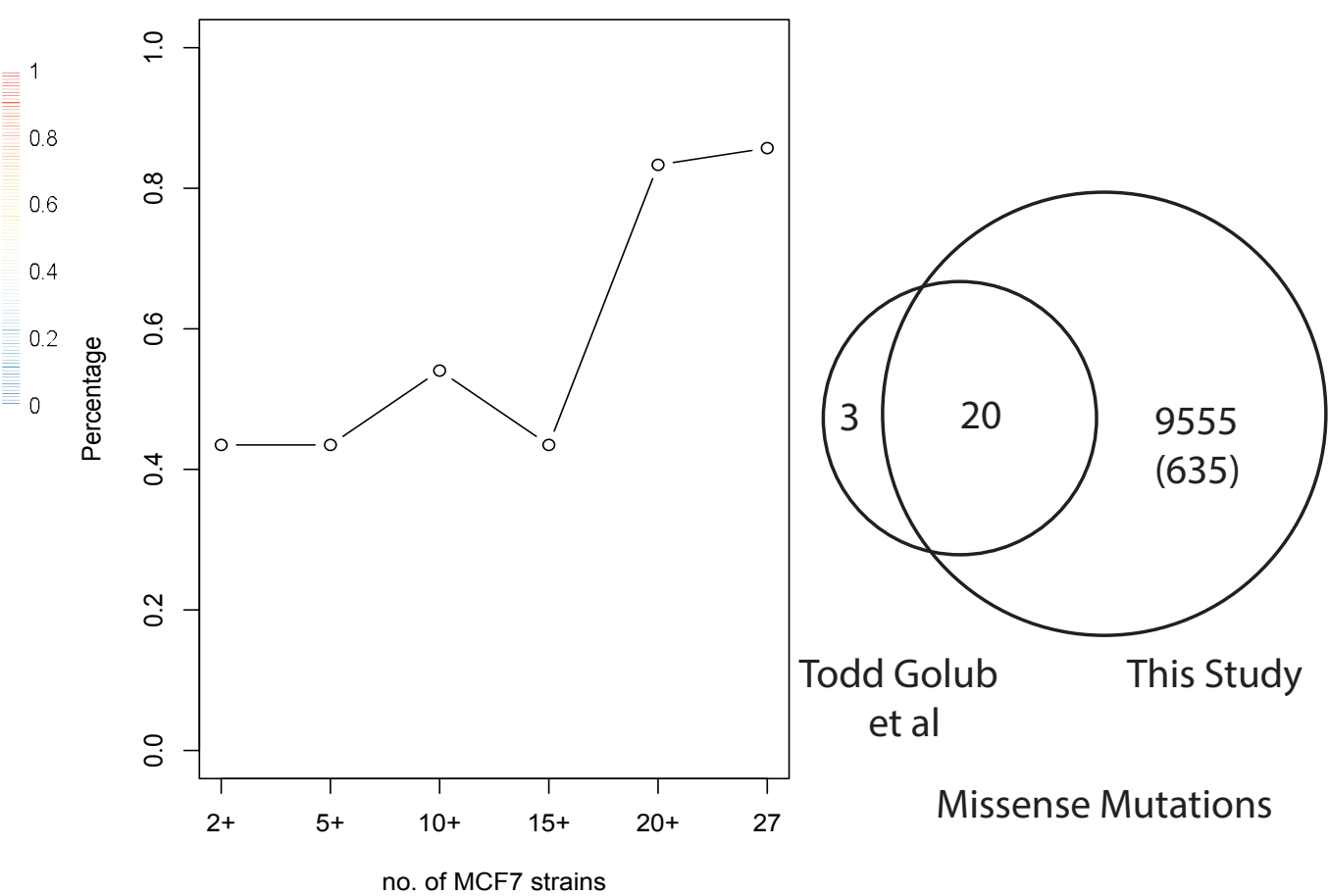

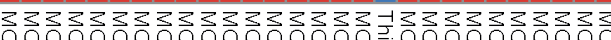

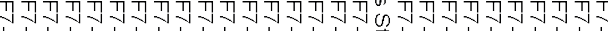

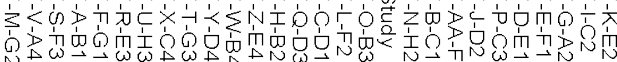

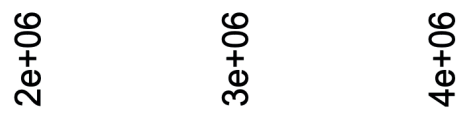

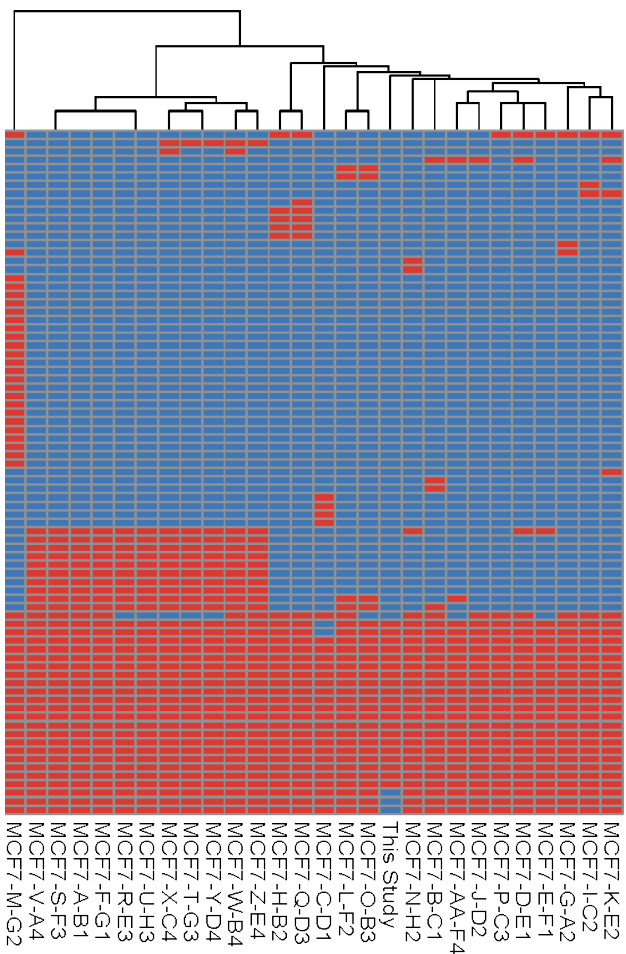


Figure 2

A
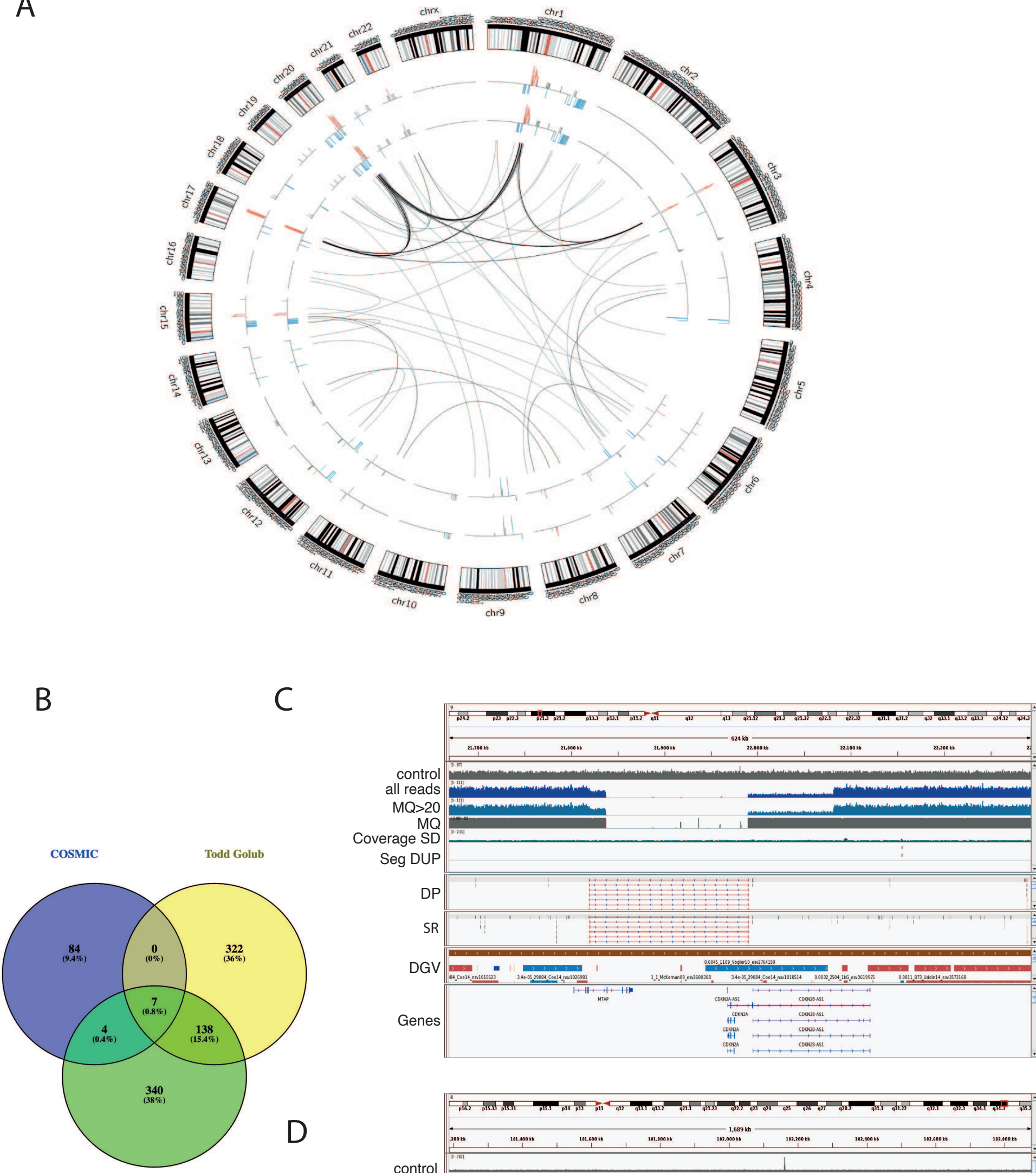

This Study

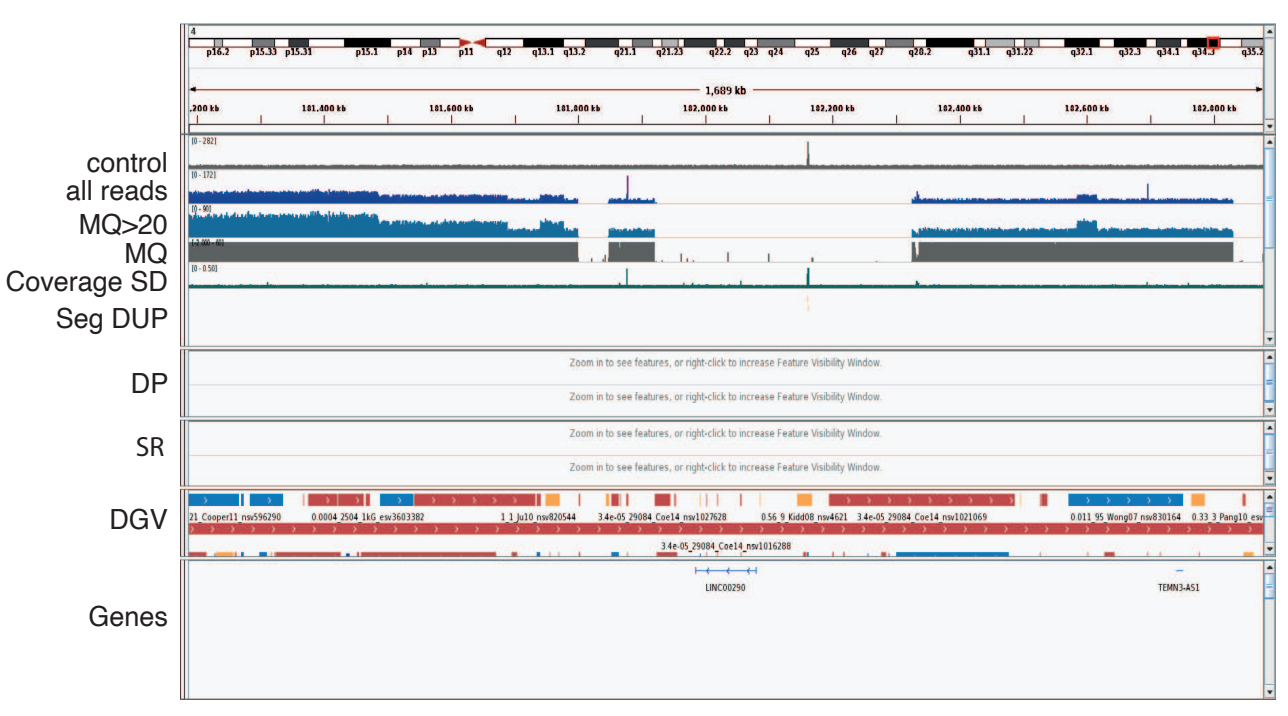


Figure 3

A
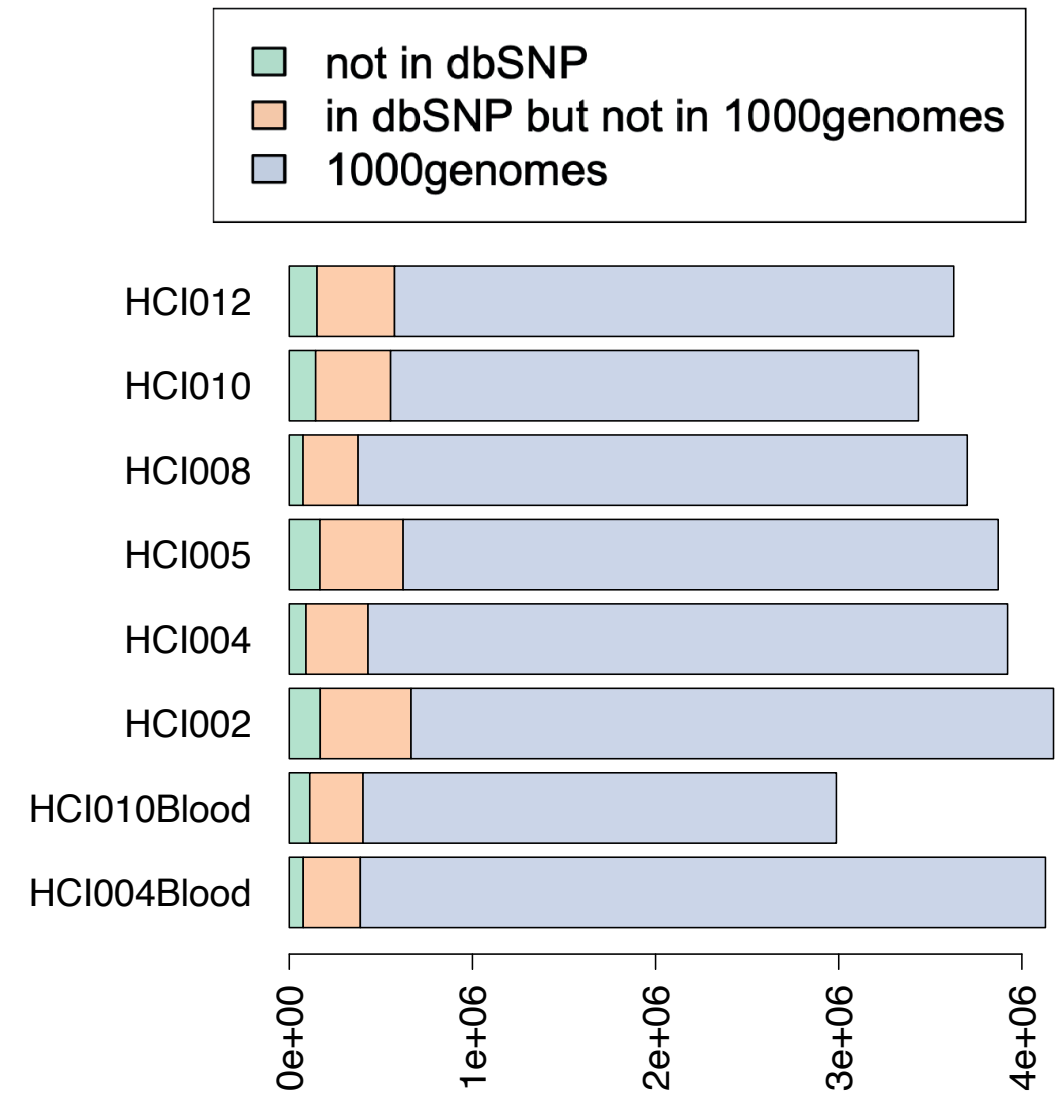

B

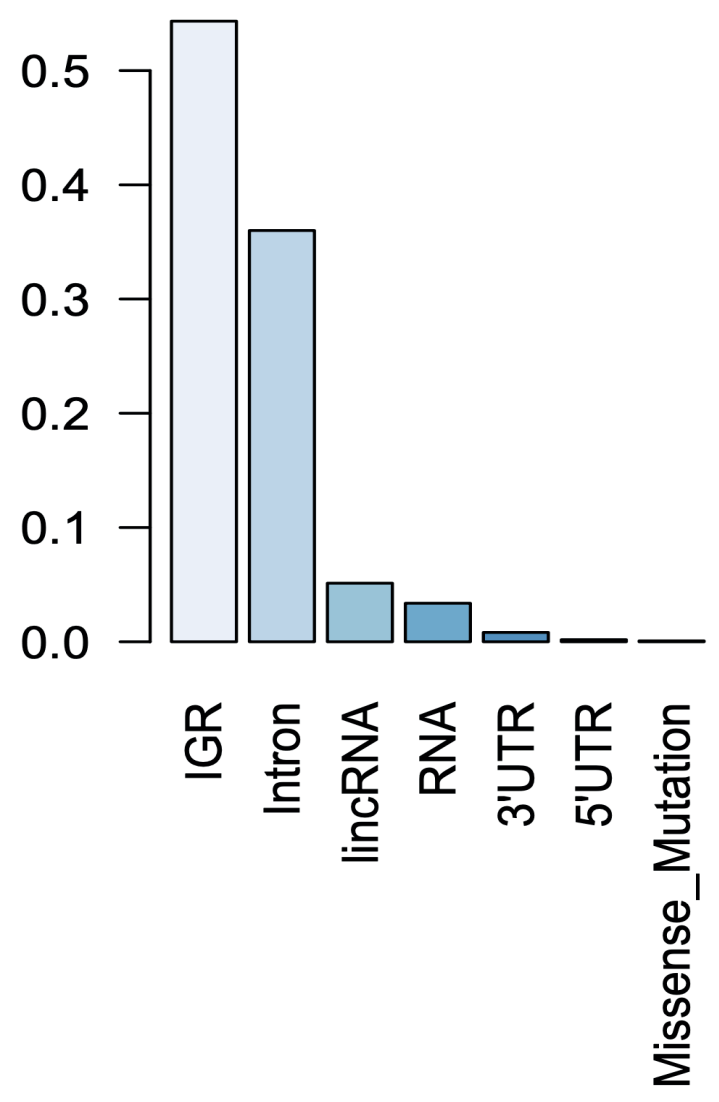

C

HCl004 germline $\mathrm{HCl004}$ tumour $M Q$ HCl004 SD SEG-DUP Genes

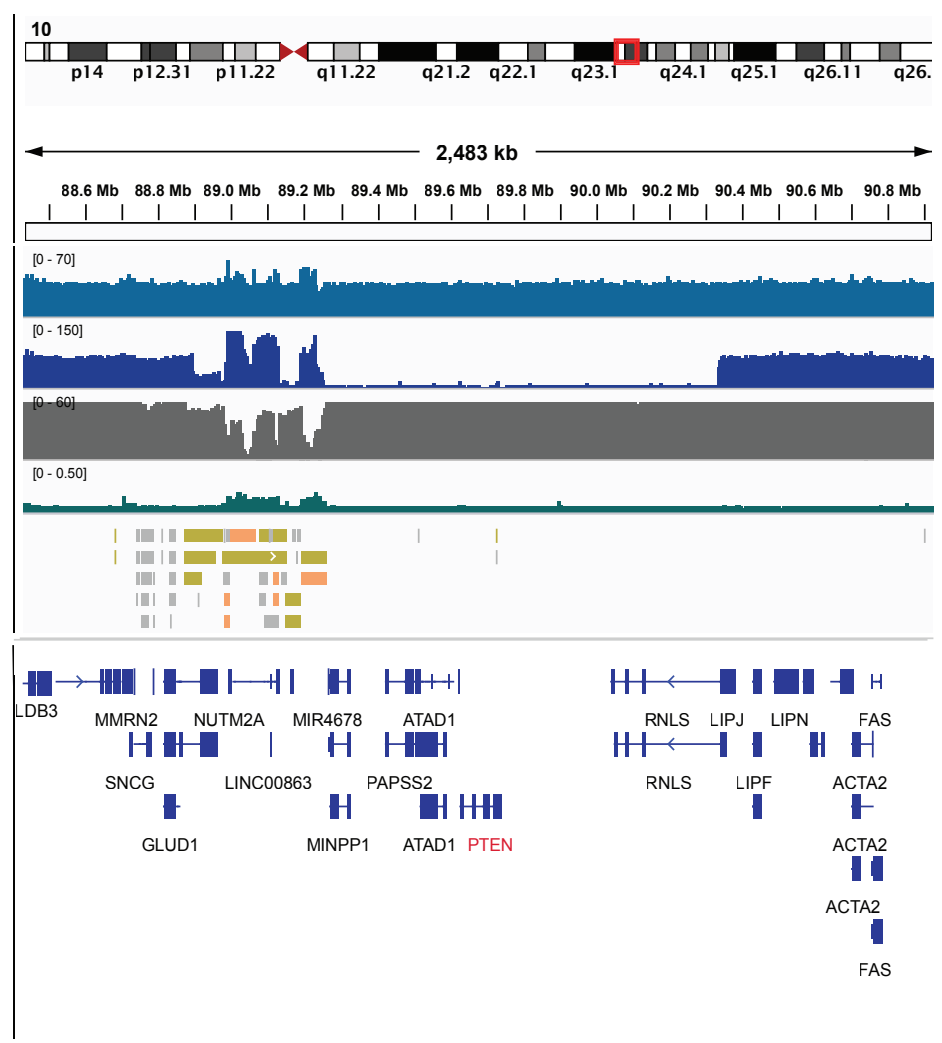


Figure 4

A. Metastatic Breast Cancer Project (cbioportal)

\begin{tabular}{|c|c|c|}
\hline \# Samples per Patient & & 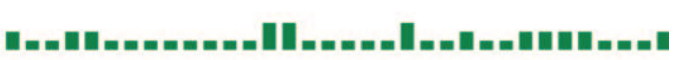 \\
\hline CSMD1 & $13 \%$ & 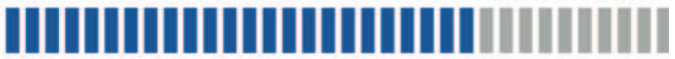 \\
\hline ZBTB16 & $6 \%$ & "III \\
\hline ONECUT2 & $4 \%$ & \\
\hline BCL2 & $4 \%$ & $\|$ \\
\hline GRP & $3 \%$ & |||||||||||||||||||||||||| \\
\hline CPLX4 & $3 \%$ & |||||||||||||||||||||- \\
\hline Genetic Alteration & & | Deep Deletion \\
\hline Samples per Patient & & \\
\hline
\end{tabular}

B. METABRIC (cbioportal)

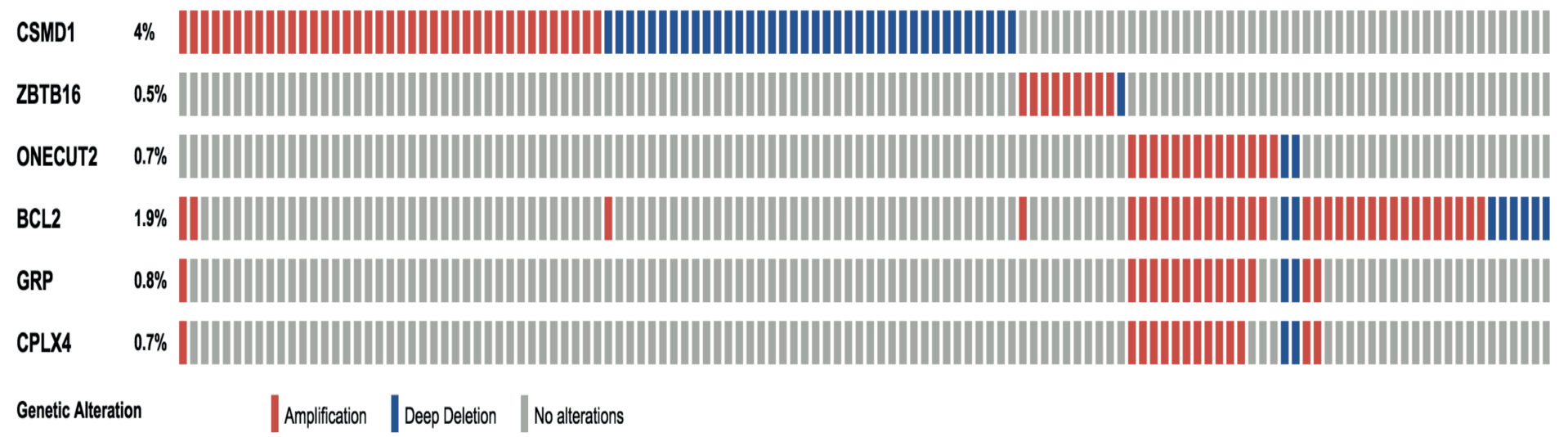

C

Survival of CSMD1 in M1 ALL $(p=0.00466)$

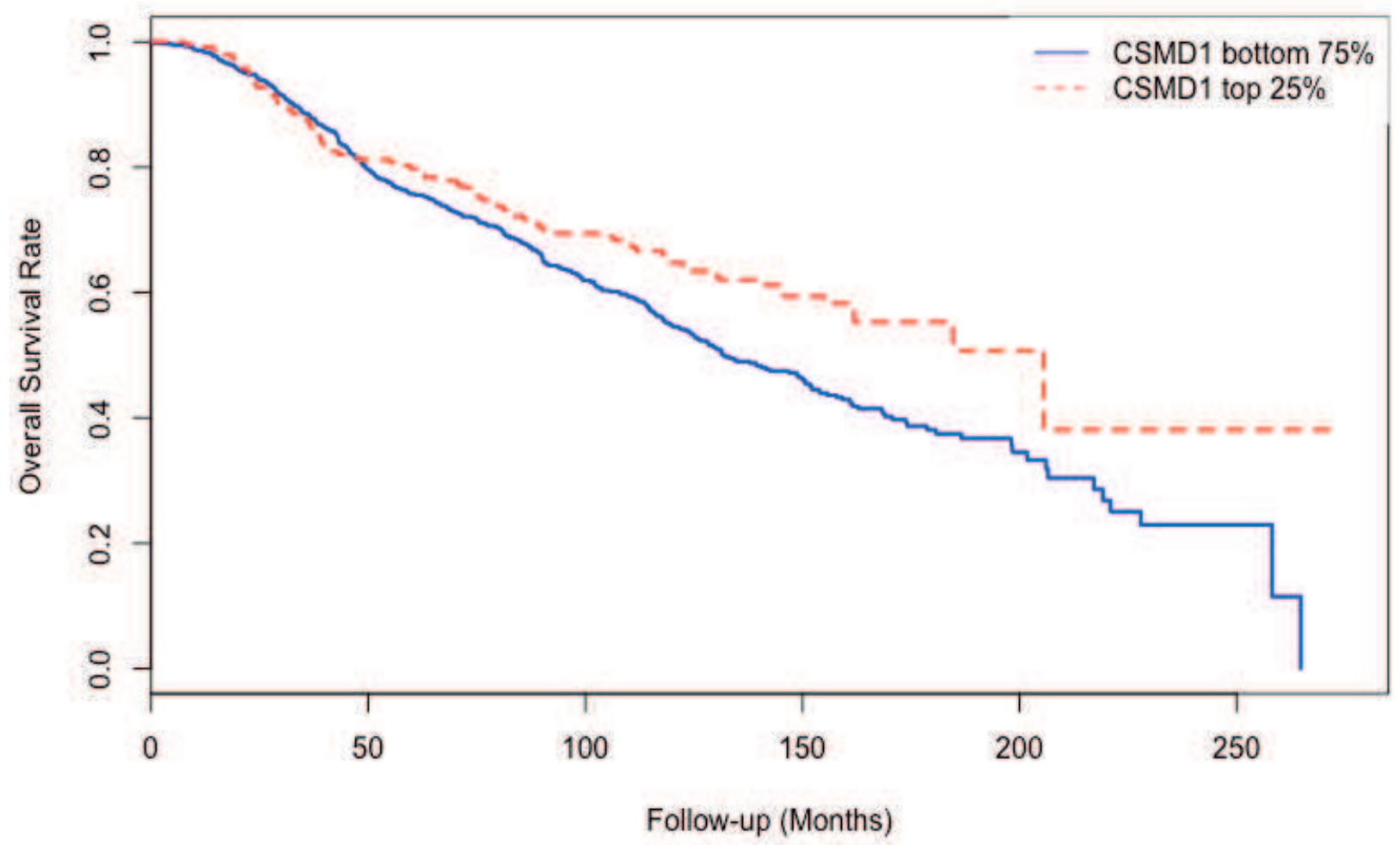


450 Deep whole genome sequencing identifies recurrent genomic alterations in breast

451 cancer cell lines and patient derived xenograft models

453 List the full names, institutional addresses and email addresses for all authors

454 Niantao Deng ${ }^{1,2}$, Andre Minoche ${ }^{2,3}$, Kate Harvey ${ }^{1}$, Meng Li ${ }^{4,5}$, Juliane Winkler ${ }^{4,5}$, 455 Andrei Goga ${ }^{4,5}$, Alex Swarbrick ${ }^{1,2}$

$456{ }^{1}$ The Kinghorn Cancer Centre \& Cancer Research Theme, Garvan Institute of 457 Medical Research, Sydney, Australia; ${ }^{2}$ St Vincent's Clinical School, Faculty of 458 Medicine, UNSW, Australia; ${ }^{3}$ Kinghorn Centre for Clinical Genomics, Garvan 459 Institute of Medical Research, Sydney, Australia; ${ }^{4}$ Department of Cell \& Tissue 460 Biology, University of California, San Francisco, San Francisco, CA, USA;

$461{ }^{5}$ Department of Medicine, University of California, San Francisco, San Francisco, 462 CA, USA.

463

464 List of supplementary figures

465

466 Figure S1: number of citations from PubMed for breast cancer cell lines.

467 Number of citations for each of the cell lines obtained from PubMed.

468 Figure S2: Heatmap of correlation of genotyping calls of breast cancer cell lines from 469 three different studies.

470 Correlation analysis of genotyping calls from this study to compare with SNP calls 471 from Heiser et al and CCLE.

472 Figure S3: IGV plot of a non-coding gene MALAT1 in MCF7 and MDAMB231

473 respectively, showing a mutation in MCF7 but not in MDAMB231 
474 Figure S4: Circos plot of structural variations in breast cancer cell lines, MDA-MB-

475 231, T47D, MDA-MB-468 and SKBR3

476 Arcs connecting two loci of difference chromosomes indicate inter-chromosomal

477 structural variations.

478 Figure S5: GREAT analysis of genes affected by SV variants in the breast cancer cell 479 lines

480 Figure S6: Representative IGV plot showing copy number gains in FOXA1, GATA3 and ESR1 in MCF7

482 Tracks from top to bottom: depth of coverage in an NA12878 control (control), all 483 reads in the sample (all reads), or reads with mapping quality $>=20(M Q>20)$, the 484 average mapping quality of aligned reads from the sample (MQ, if no reads align $485 M Q=0$ ), coverage standard deviation from 500 controls (Coverage $S D$, indicating common $C N V$ ), overlapping segmental duplications published by Bailey JA et al. 2002 (SEG-DUP, used as control for germline CNVs), discordant pairs (DP), split 488 reads (SR), variants from the Database of Genomic Variants (DGV), and RefSeq 489 genes (Genes).

491 List of supplementary tables

492 Table S1-S11

493 Table S1: Summary of total number of variants in difference cell line models and

494 PDXs from whole genome sequencing

495 Table S2: List of the 635 additional missense mutations in MCF7 compared to Ben-

496 David et al

497 Table S3: The complete list of variants in the non-coding regions in these cell lines 498 models 
499 Table S4: List of structural variants identified in the cell line models

500 Table S5: List of copy number alterations identified in the cell line models

501 Table S6: Comparison of the genes with copy number alterations in COSMIC, Ben-

502 David et al and this study

503 Table S7: Summary of sequencing depth for PDXs

504 Table S8: List of mutations identified and validated in breast cancer PDXs

505 Table S9: List of copy number alterations and identified in the PDXs

506 Table S10: List of structural variants identified in the PDXs

507 Table S11: List of genes with common copy number alterations in the PDXs 
Figure S1

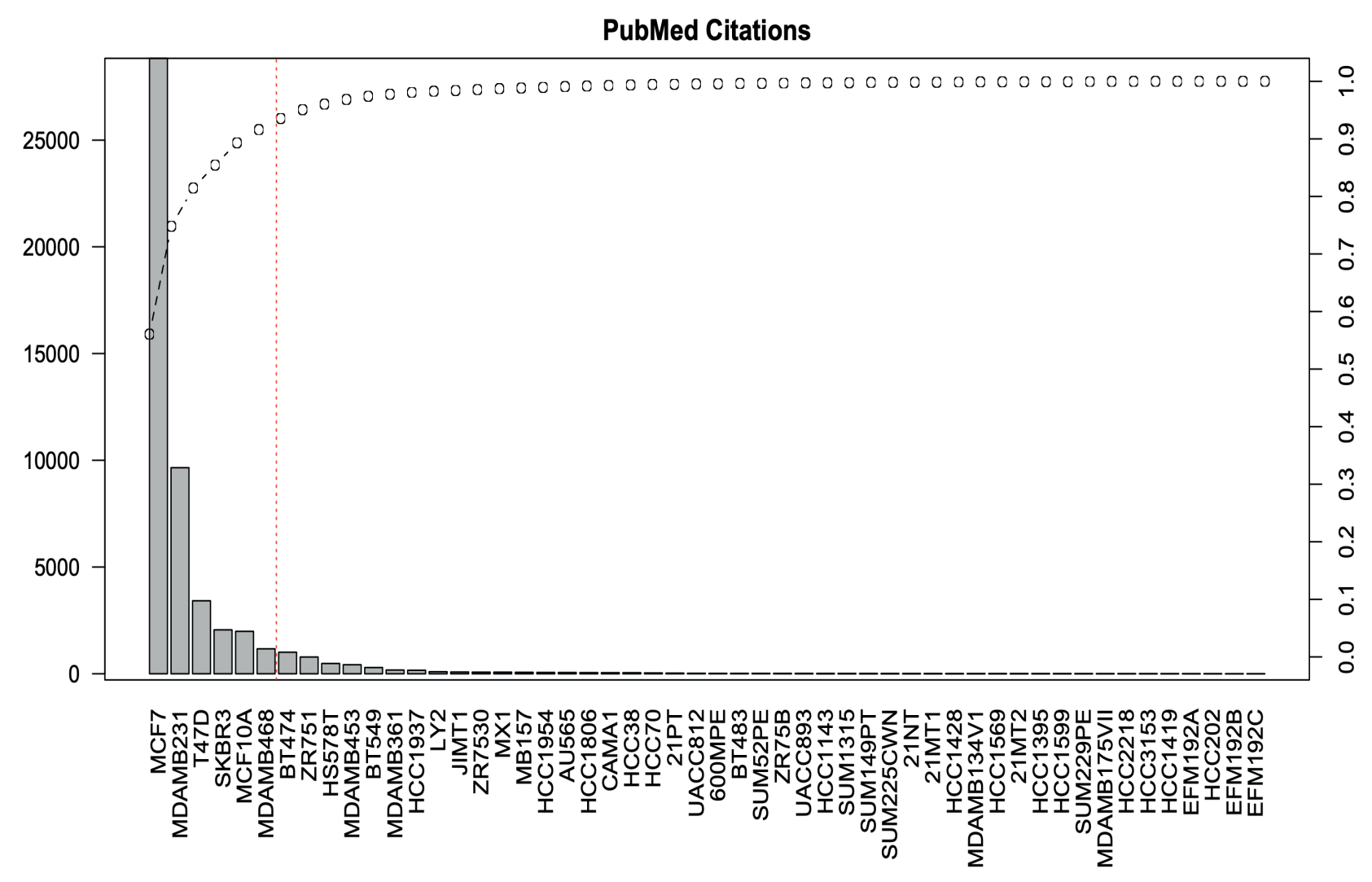

Figure S2

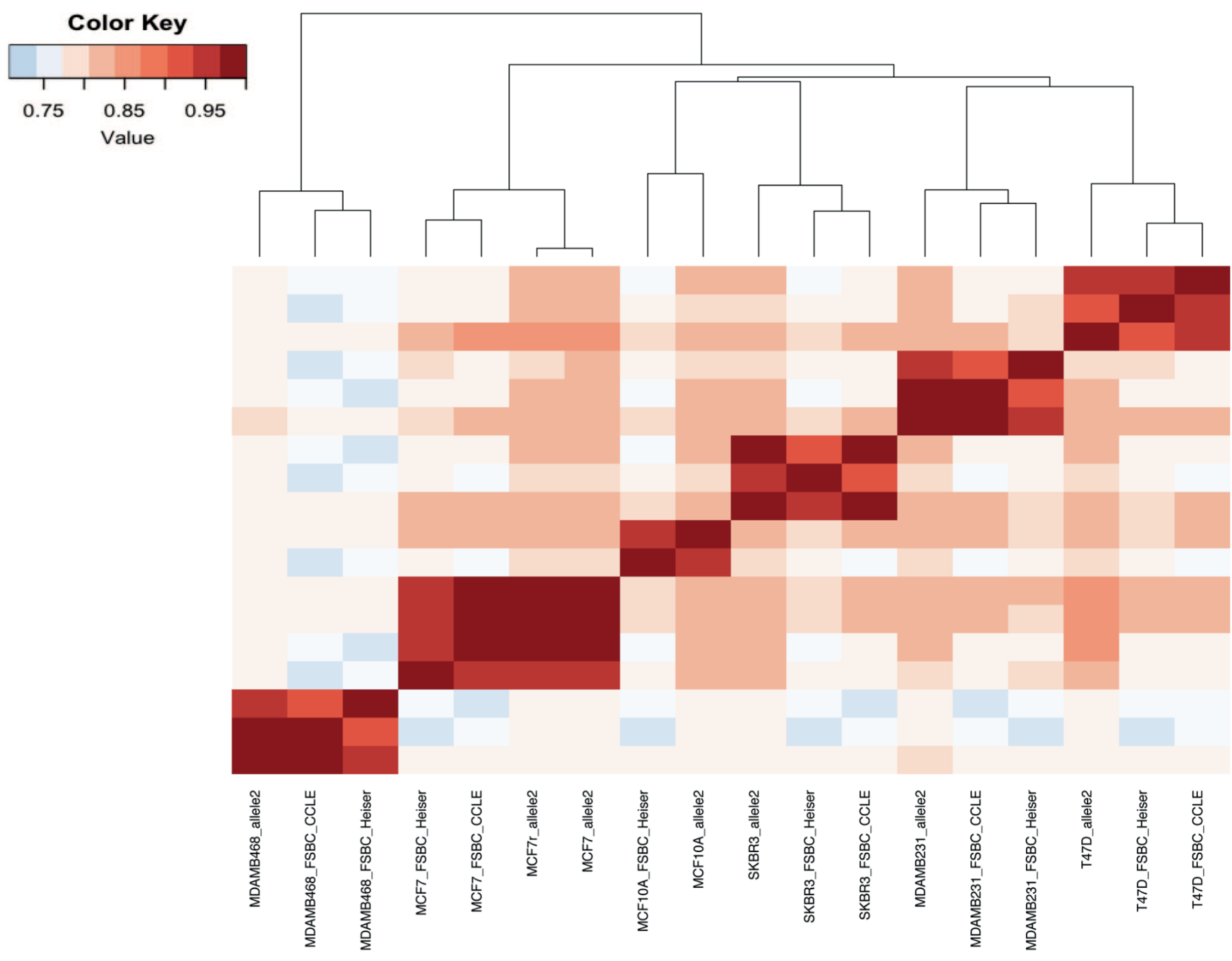


Figure S3

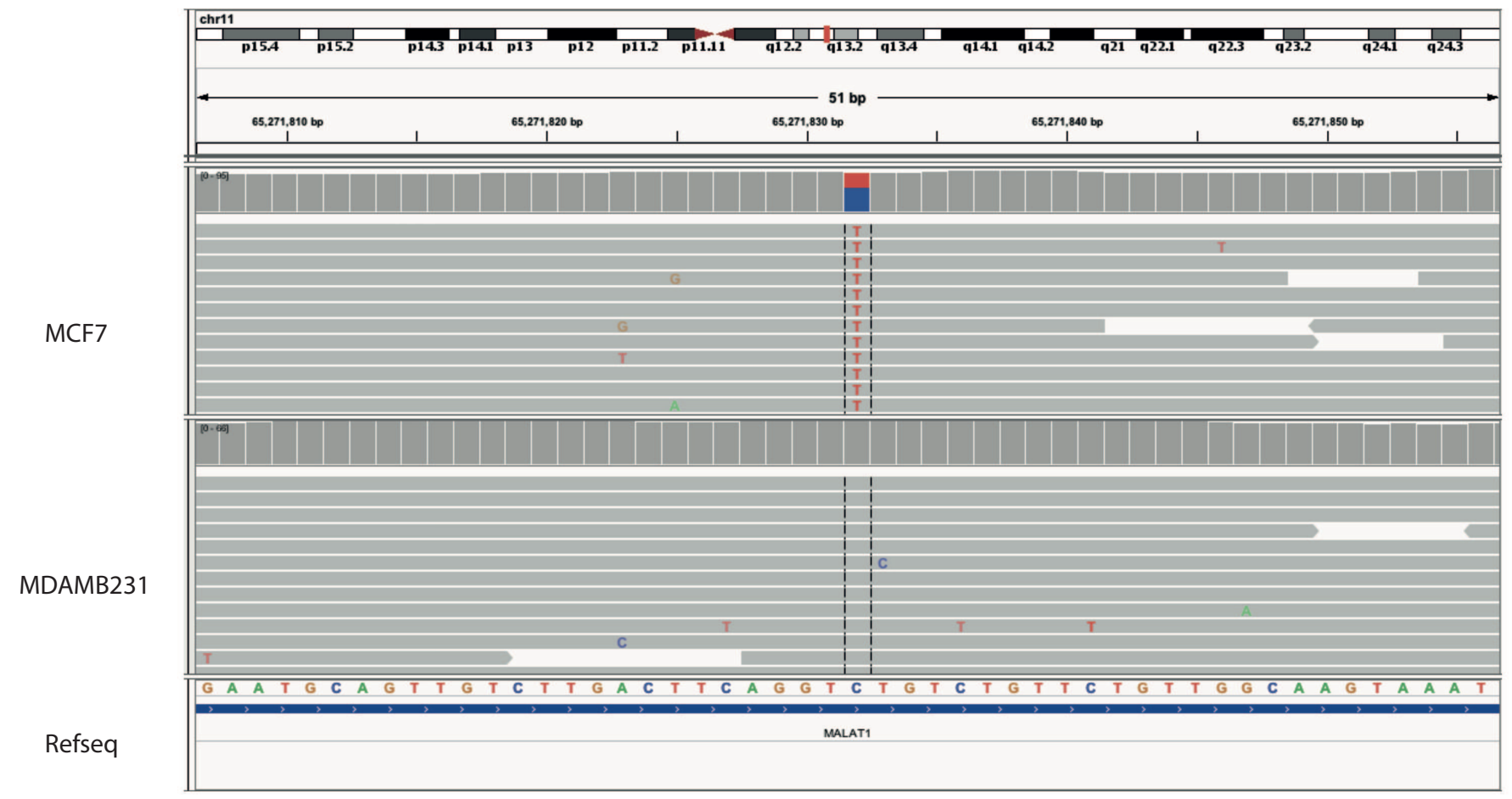

Figure S4 MDA-MB-231
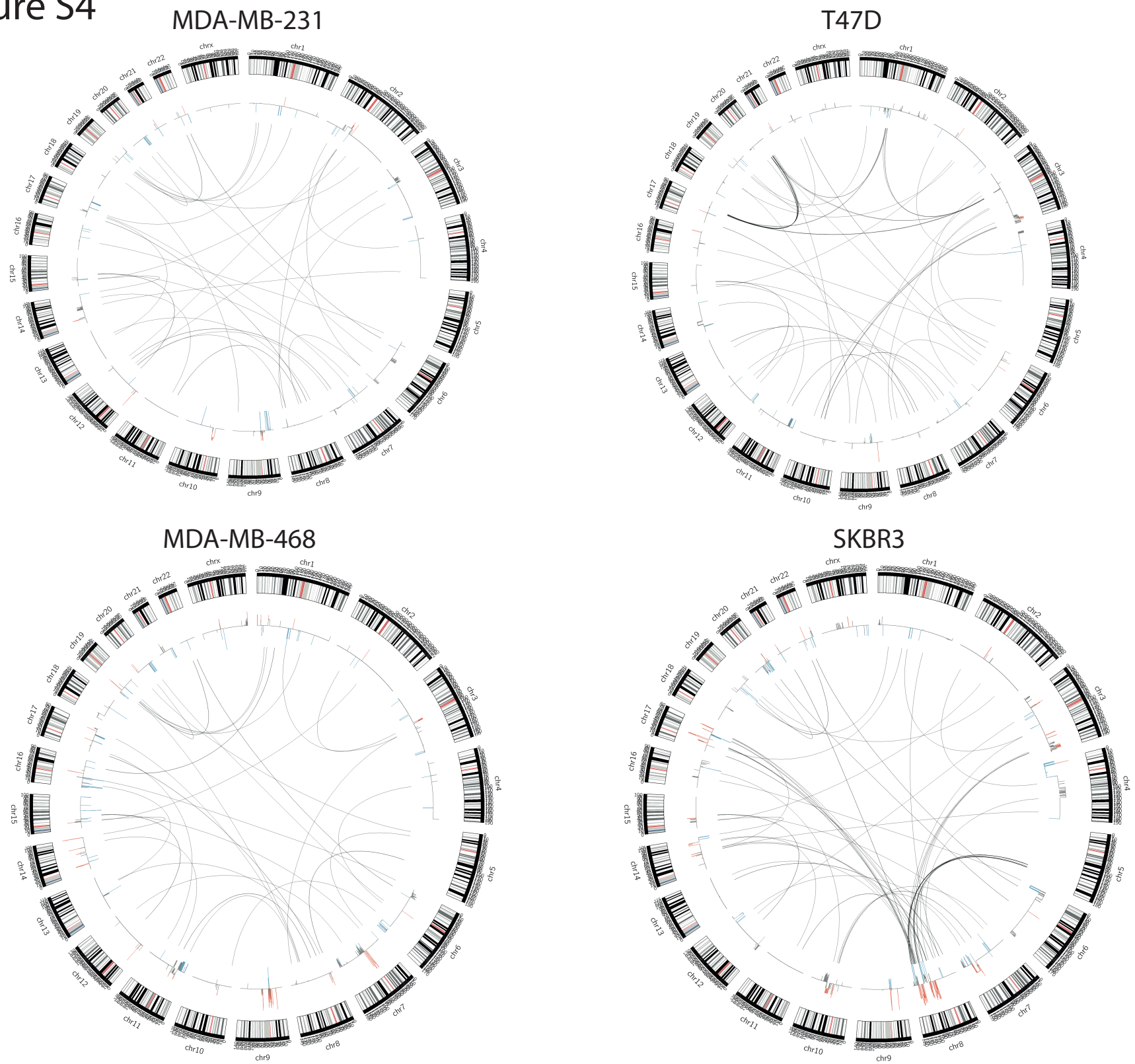


\section{Figure S5}

\section{MSigDB Perturbation}

\section{MCF7}

- $\log 10$ (Binomial $p$ value)

Genes within amplicon 20q12-q13 identified in 191 breast tumours .

Genes within amplicon 17q21-q25 identified in 191 breast tumours .

$\begin{array}{lllllllllll}0 & 5 & 10 & 15 & 20 & 25 & 30 & 35 & 40 & 45 & 50\end{array}$

Cluster 6: selected luminal genes clustered across breast tumours

10.56

\section{MSigDB Perturbation}

T47D

$-\log 10$ (Binomial p value)

\section{0}

Genes within amplicon 20q12-q13 identified in 191 breast tumours Genes within amplicon 17q21-q25 identified in 191 breast tumours Cluster 6: selected luminal genes clustered across breast tumours Genes up-regulated with HEMGN in the TLX1 Tet on iEBHX15-4 cells.

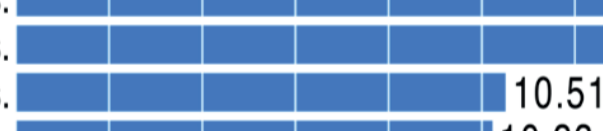

\section{MSigDB Perturbation}

\section{SKBR3}

Genes within amplicon 8q23-q24 identified in 191 breast tumours Genes down-regulated in HFK cells in response to UVB irradiation Genes with putative STAT5 Genes up-regulated by IL2

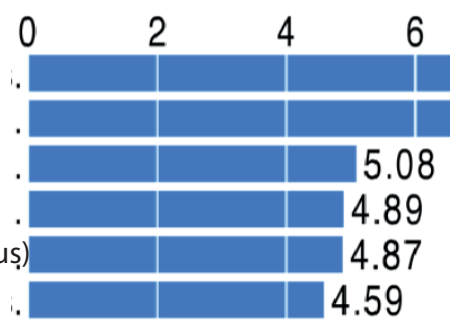




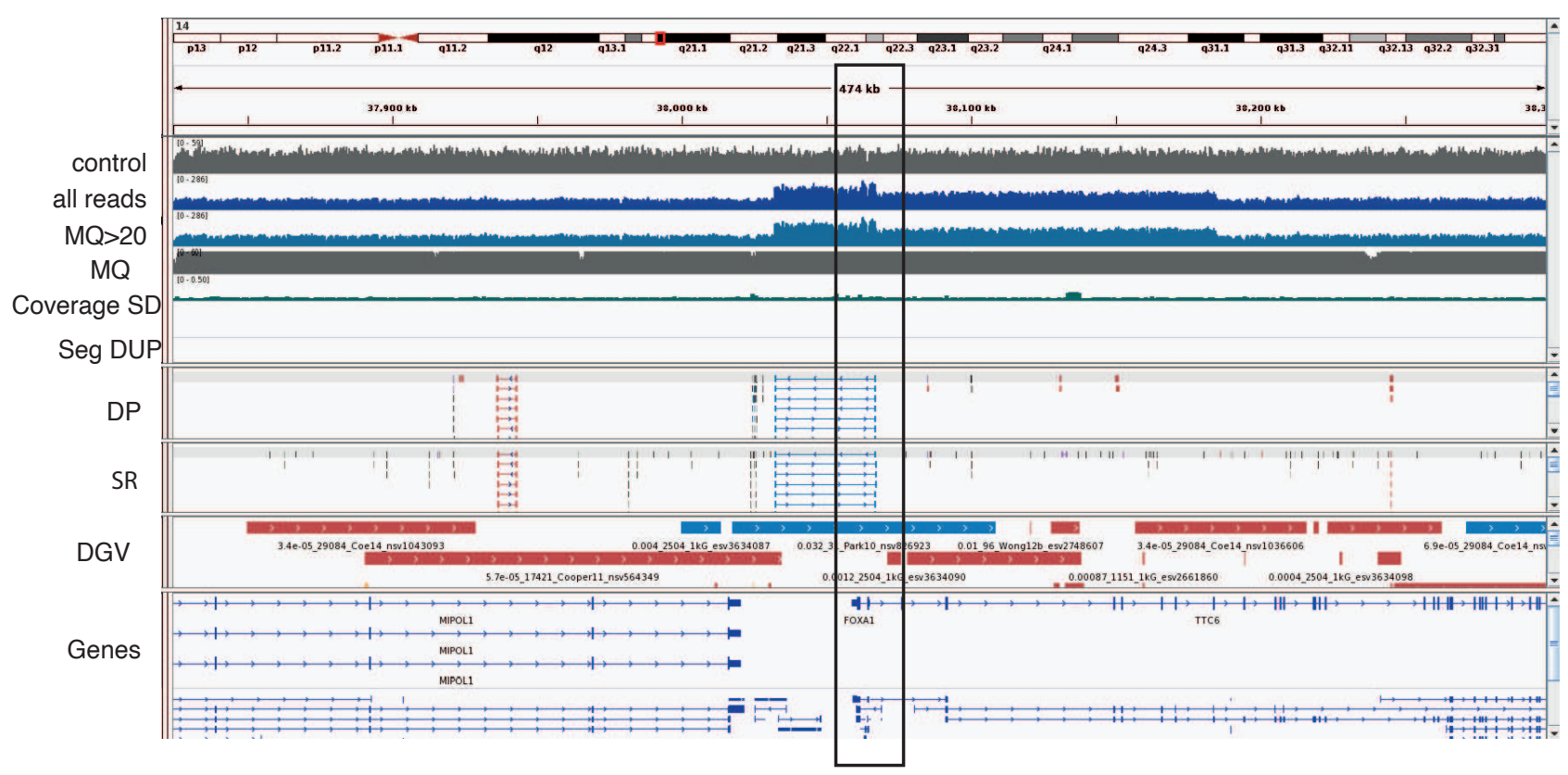

GATA3, broad gain, 4 copies
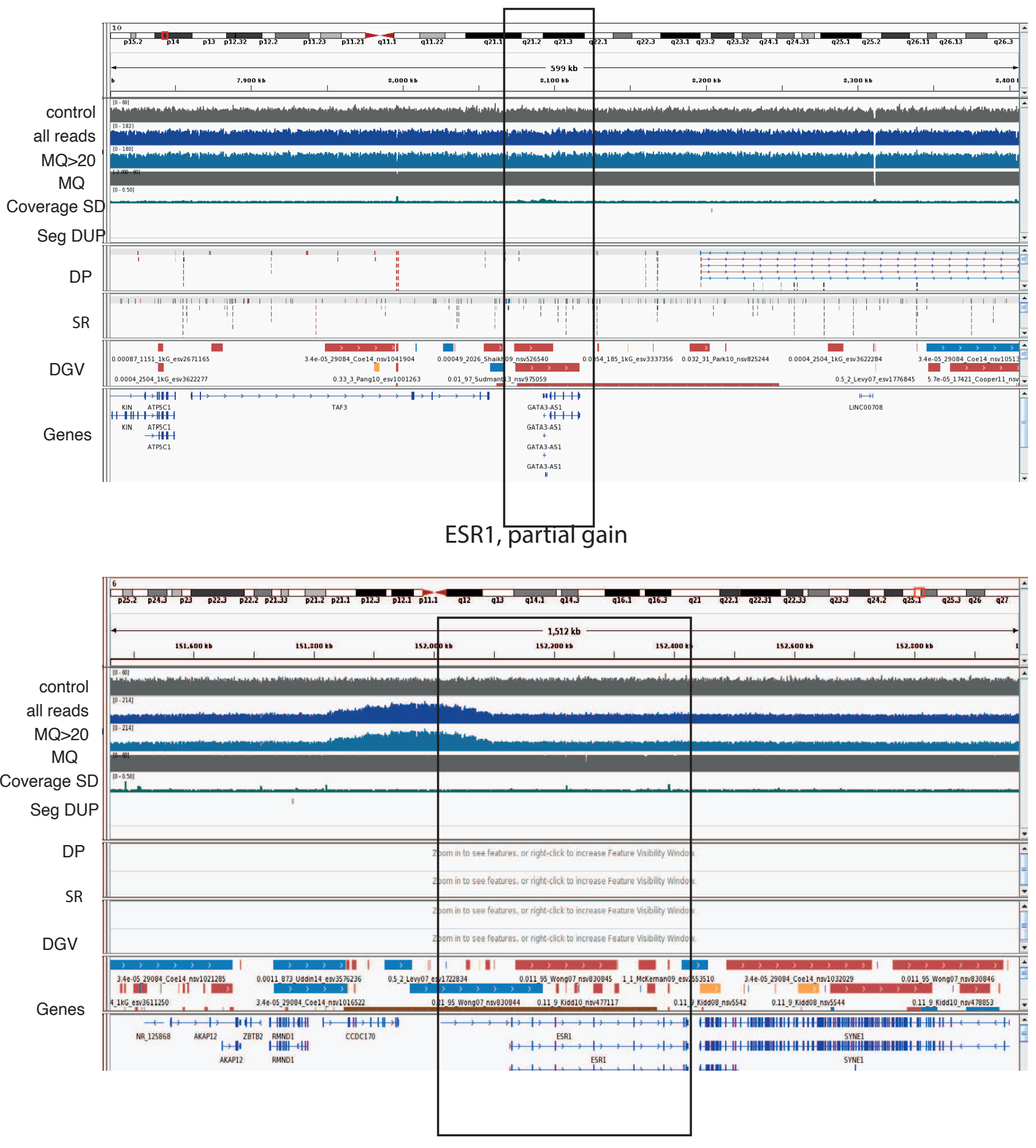


\section{Supplementary Files}

This is a list of supplementary files associated with this preprint. Click to download.

- SuppTable14.xlsx

- SuppTableS5.xIsx

- SuppTableS6.xlsx

- SuppTables78.xlsx

- SuppTableS9.xlsx

- SuppTable10.xlsx

- SuppTableS11.xlsx 